\title{
A fully covered self-expandable metal stent anchored by a 10-Fr double pigtail plastic stent: an effective anti-migration technique
}

\section{Panagiotis Katsinelos ${ }^{a}$, Georgia Lazarakia, Stergios Gkagkalisa, Grigoris Chatzimavroudis ${ }^{a}$, Kiriaki Anastasiadou ${ }^{b}$, Nikos Georgakis ${ }^{b}$, Olga Gioulemea ${ }^{a}$, Christos Zavos ${ }^{b}$, Jannis Kountouras ${ }^{b}$}

G. Gennimatas General Hospital, Medical School, Aristotle University of Thessaloniki; Ippokration Hospital, Medical School, Aristotle University of Thessaloniki, Thessaloniki, Greece

\section{Abstract}

Background Fully covered self-expandable metal stents (FCSEMS) have been used successfully in the treatment of malignant and benign biliary strictures. However, stent migration is a major complication. We investigated the efficacy of anchoring FCSEMS with a 10-Fr double-pigtail plastic stent to prevent migration in patients with biliary strictures.

Methods Between January 2012 and May 2013, 10 patients with malignant biliary strictures and one patient with a suprapapillary benign biliary stenosis were enrolled in the study. The primary endpoint of the study was to record the migration rate of FCSEMS.

Results The placement of FCSEMSs and the anchoring with a 10-Fr double-pigtail plastic stent were successful in all patients. During a median follow-up period of eight months, proximal or distal migration of FCSEMS was not observed. No procedural complications related to the placement of FCSEMS and/or the anchoring plastic stent were recorded.

Conclusions The placement of an anchoring 10-Fr double-pigtail stent is a simple and effective anti-migration technique for FCSEMS in patients with malignant biliary strictures.

Keywords Fully covered self-expandable metal stents, migration, prevention

Ann Gastroenterol 2017; 30 (1): 1-4

\section{Introduction}

Uncovered self-expandable biliary metal stents were a revolution in the treatment of malignant biliary strictures. In contrast to plastic stents, uncovered metallic stents maintain lumen patency for a significantly longer period, reducing the

${ }^{a}$ Department of Endoscopy and Motility Unit, G. Gennimatas General Hospital, Medical School, Aristotle University of Thessaloniki (Panagiotis Katsinelos, Georgia Lazaraki, Stergios Gkagkalis, Grigoris

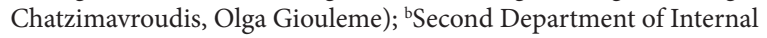
Medicine, Ippokration Hospital, Medical School, Aristotle University of Thessaloniki (Kiriaki Anastasiadou, Nikos Georgakis, Christos Zavos, Jannis Kountouras), Thessaloniki, Greece

Conflict of interest: None

Correspondence to: Dr. Panagiotis Katsinelos, Assoc. Professor of Gastroenterology, Head, Department of Endoscopy and Motility Unit, G. Gennimatas General Hospital, Medical School, Aristotle University of Thessaloniki, Ethnikis Aminis 41, Thessaloniki, Greece, Tel.: +30 2310 963341, Fax: +302310 210401, e-mail: gchatzimav@yahoo.gr

Received 25 April 2016; accepted 27 August 2016; published online 22 September 2016

DOI: http://dx.doi.org/10.20524/aog.2016.0089 ultimate financial cost per patient [1]. However, after a while, tumor ingrowth and epithelial hyperplasia through the cells of the uncovered stent mesh occur, leading to obstruction of the stent's lumen. Thus, as it becomes difficult to remove uncovered stents endoscopically, they are not appropriate for the treatment of benign biliary strictures [2-4]. Compared with uncovered self-expandable biliary metal stents, fully covered self-expandable metal stents (FCSEMS) have longer patency and a lower rate of tumor ingrowth; however, migration has been reported to occur in up to $37.5 \%$ of cases [1].

In this study we describe our experience with an effective anti-migration technique that includes the placement of a 10-Fr double-pigtail plastic stent within the FCSEMS in a series of patients with strictures of the common bile duct (CBD).

\section{Patients and methods}

Between January 2012 and May 2013, after permission for the use of FCSEMS had been granted by the Financial Committee of our hospital, 11 patients with a CBD stricture (of malignant origin in 10 patients and benign in 1) were prospectively enrolled to receive an FCSEMS and a 10-Fr double-pigtail plastic stent within the FCSEMS to anchor it. The Institutional Review 
Board and the Ethics Committee of our hospital approved the study protocol. Written informed consent was obtained from all patients. All endoscopic procedures were performed by one experienced endoscopist (PK) who performs more than 200 pancreaticobiliary endoscopic procedures per year on an inpatient basis. After an overnight fast, all patients underwent the procedure in the prone or semiprone position with a therapeutic duodenoscope (TJF-180, Olympus, Tokyo, Japan). Patients' blood pressure, pulse rate and oxygen saturation were under continuous monitoring during the procedure. Conscious sedation was achieved using 25-50 mg intravenous pethidine hydrochloride and midazolam $(0.05 \mathrm{mg} / \mathrm{kg})$. Hyoscine-n-butyl (Buscopan, Boehringer Ingelheim, Ltd., UK) or glucagon was used as smooth muscle relaxant, at the endoscopist's discretion. For ductal opacification, a solution of contrast medium (50\% sodium meglumine amide triazoate) diluted in distilled water and impregnated with ciprofloxacin $(100 \mathrm{mg}$ per $50 \mathrm{~mL}$ contrast medium) was used.

In patients who had not previously undergone endoscopic retrograde cholangiopancreatography (ERCP), sphincterotomy was performed after biliary cannulation via a hydrophilic guidewire (Dreamwire, Boston Scientific, Athens, Greece). Routine biopsy from ampullary tumors or transpapillary brush cytology was performed, as well as biopsy of the distal biliary stricture for confirmation of malignancy. The proximal end of the super stiff hydrophilic guidewire (Jagwire, Boston Scientifc, Athens, Greece) was placed high in the liver. The length of the biliary stricture was measured and the FCSEMS delivery system (Wallflex, Boston Scientific, Athens, Greece) was advanced over the guidewire, with the proximal end of the stent placed above the malignant stricture or tumor ingrowth and the distal end across the papilla or the distal end of any previously placed uncovered metal stent. FCSEMS of 40 or $60 \mathrm{~mm}$ were deployed. A 10-Fr 7-cm double-pigtail plastic stent (Wallstent, Boston Scientific, Athens, Greece) was then immediately inserted within the FCSEMS along the stiff guidewire. The proximal end of the anchoring stent was positioned in the bile duct over the proximal end of the FCSEMS. The distal pigtail was released halfway through to fix the distal end of the FCSEMS firmly to the duodenal mucosa. Adverse events were assessed according to established criteria.

Liver function and serum pancreatic enzyme tests were performed before stent placement, and 1 and 2 days after stent placement. An abdominal radiograph was obtained after stent placement to identify the position of the stent. During the follow-up period, patients were seen in the outpatient clinic at 1-month intervals, each time with a plain abdominal radiograph to verify the stent position. Liver serum function tests were also performed. Proximal stent migration was defined as any migration of the FCSEMS into the bile duct and distal stent migration was classified as spontaneous migration (even without the stent becoming lodged in the bowel or impacting on the duodenal wall opposite the papilla).

The primary endpoint of the study was to investigate the migration rate of FCSEMS after anchoring with the 10-Fr 7-cm double-pigtail plastic stent. A secondary endpoint was procedural complications related to FCSEMS placement.

\section{Results}

Eleven patients ( 3 men, 8 women; median age 83 years, range $72-87$ ) were treated using the anti-migration technique at our center (Table 1). In 6 patients the obstruction was due to tumor ingrowth in an uncovered metal stent placed previously for a malignant stricture of the CBD (Table 1). In 4 patients, 2 with ampullary cancer and 2 with pancreatic cancer, we used an FCSEMS as first choice of treatment (Table 1). One patient presented a suprapapillary stenosis as a late complication of post-endoscopic sphincterotomy; multiple unsuccessful efforts to dilate the stenosis using a $\mathrm{CRE}^{\text {tax }}$ balloon (diameter $12 \mathrm{~mm}$ ) and placement of multiple plastic stents proved the stenosis to be refractory. The FCSEMS and anchoring plastic stent were inserted successfully in all patients.

The median follow-up period was eight months (range 4-14 months) and no migration of FCSEMS was observed. None of the patients presented post-ERCP complications or other complications directly related to FCSEMS placement.

\section{Discussion}

FCSEMSs have a complication rate that ranges between $0 \%$ and $45 \%$, with the most common being proximal or distal stent migration, periprocedural pancreatitis, acute cholecystitis and secondary CBD strictures [1-4]. Two antimigration modifications, anchoring fins and flared ends, have been introduced to the market $[5,6]$. There are no randomized studies comparing stent migration in FCSEMS with and without fins and flared ends. However, studies have shown that FCSEMS with anchoring fins have relatively lower rates of stent migration (0.7-7\%), at the expense of notable rates of mucosal ulcerations and bleeding during stent removal [6]. The long-term sequelae of these ulcerations are currently unknown.

Recently, Park et al [7] described a technically simple and effective anti-migration technique. In a randomized controlled study they placed FCSEMS in 33 patients with benign biliary strictures. In 17 patients, after deployment of the stent in the usual fashion, they placed a 5-Fr plastic double-pigtail stent through the FCSEMS over the same guidewire used for FCSEMS deployment (anchored group). The proximal pigtail was laid in the right or left main hepatic duct while the distal end was allowed to curl around the edge of the FCSEMS and into the duodenum. During follow up, significantly fewer patients who underwent FCSEMS placement with an anchoring stent presented migration compared to patients in the non-anchored group: $1 / 16(6.3 \%)$ vs. $7 / 17$ (41.2\%), $\mathrm{P}=0.024$.

Our present series is different from the study of Park et al because we included mainly patients with ampullary carcinoma (6 patients), distal CBD cancer (2 patients) and pancreatic cancer ( 2 patients), and only one with a benign biliary stricture. In 6 of these an uncovered metal stent had been placed in other hospitals and the patients were referred 
Table 1 Results of placing a 10-Fr double-pigtail plastic stent within an FCSEMS as an anti-migration technique

\begin{tabular}{|c|c|c|c|c|c|c|}
\hline Patients & Sex & Age & Reason for FCSEMS placement & $\begin{array}{l}\text { Previous uncovered } \\
\text { stent placement }\end{array}$ & $\begin{array}{l}\text { FCSEMS } \\
\text { migration }\end{array}$ & $\begin{array}{l}\text { Follow up } \\
\text { (months) }\end{array}$ \\
\hline 1 & $\mathrm{~F}$ & 82 & Tumor ingrowth of ampullary cancer & Yes & No & 14 \\
\hline 2 & M & 81 & Tumor ingrowth of distal CBD cancer & Yes & No & 9 \\
\hline 3 & M & 79 & Tumor ingrowth of ampullary cancer & Yes & No & 10 \\
\hline 4 & $\mathrm{~F}$ & 85 & Tumor ingrowth of ampullary cancer & Yes & No & 7 \\
\hline 5 & M & 83 & Tumor ingrowth of distal CBD cancer & Yes & No & 8 \\
\hline 6 & M & 87 & Tumor ingrowth of ampullary cancer & Yes & No & 4 \\
\hline 7 & M & 83 & Ampullary cancer & No & No & 8 \\
\hline 8 & M & 81 & Ampullary cancer & No & No & 8 \\
\hline 9 & M & 83 & Pancreatic cancer & No & No & 5 \\
\hline 10 & $\mathrm{~F}$ & 84 & Pancreatic cancer & No & No & 5 \\
\hline 11 & M & 72 & Suprapapillary stenosis after sphincterotomy & No & No & 10 \\
\hline
\end{tabular}

FCSEMS, fully covered self-expanding metal stent; $F$, female; $M$, male; $C B D$, common bile duct

to our unit for stent obstruction due to tumor ingrowth. It is well known that ampullary and biliary cancer of the two lower thirds of the CBD are adenocarcinomas with an exophytic appearance and present high ingrowth rate in uncovered metal stents. In some patients who have previously undergone placement of an uncovered self-expanding metal stent and subsequently exhibit tumor ingrowth, when the obstruction is not complete the pressure exerted on the wall of the FCSEMS can be lower than the pressure exerted by a refractory benign biliary stricture, resulting in a higher migration rate [1-3]. It is notable that the use of a 10-Fr plastic stent in our patients (twice the diameter of the 5-Fr plastic stent in the study of Park et al) increased the strength of the anchoring mechanism and minimized the migration rates.

Acute cholecystitis after placement of a biliary FCSEMS has been reported in up to $12 \%$ and involvement of the cystic orifice by tumor is a major risk factor for occurrence of cholecystitis [8-10]. None of our patients developed acute cholecystitis after FCSEMS placement. The absence of this complication in our study is related to the fact that in our patients, especially those with ampullary carcinoma, the cystic duct orifice was free from tumor infiltration. It has been demonstrated that incomplete biliary drainage is predictive of $91 \%$ of all cases of sepsis [11]. In addition, our policy of using ciprofloxacin-impregnated contrast material during ERCP for the last 5 years has been proved effective. Local injection of antibiotics along with the non-sterile contrast material during biliary opacification is based on the rationale of lock therapy, as used for the sterilization of central iv catheters [12], and has been particularly useful in manipulation of the biliary tree during ERCP in our practice up to now. Future studies investigating the local use of antibiotics in this patient population are warranted. Other procedural complications related to FCSEMS and anchoring plastic stent placement were not observed in this series.

Limitations of the present study are the small sample size and the fact that it was not randomized. However, this is the first study to examine the effectiveness of placing a 10-Fr double-pigtail plastic stent inside an FCSEMS as an anchoring mechanism in a variety of biliary strictures (mostly malignant) as an anti-migration technique.

In conclusion, the placement of a 10-Fr plastic pigtail biliary stent inside an FCSEMS as an anchoring mechanism is a simple and effective anti-migration method.

\section{Summary Box}

\section{What is already known:}

- Self-expandable biliary metal stents (SEMS) are effective for the treatment of malignant biliary strictures, maintaining lumen patency for a significantly longer period than do plastic stents

- Uncovered SEMS bear the limitation of tumor ingrowth and epithelial hyperplasia through the mesh cells, leading to obstruction of the stent's lumen

- Fully covered SEMS (FCSEMS) have longer patency and a lower rate of tumor ingrowth; however, migration has been reported to occur in up to $37.5 \%$

\section{What the new findings are:}

- Placing a 10-Fr double-pigtail plastic stent inside an FCSEMS is a feasible and effective antimigration technique

- Although an FCSEMS was used, in our patients we did not observe any episodes of post-endoscopic retrograde cholangiopancreatography cholecystitis or cholangitis 


\section{References}

1. Dumonceau JM, Tringali A, Blero D, et al; European Society of Gastrointestinal Endoscopy. Biliary stenting: indications, choice of stents and results: European Society of Gastrointestinal Endoscopy (ESGE) clinical guideline. Endoscopy 2012;44:277-298.

2. Saleem A, Leggett CL, Murad MH, Baron TH. Meta-analysis of randomized trials comparing the patency of covered and uncovered self-expandable metal stents for palliation of distal malignant bile duct obstruction. Gastrointest Endosc 2011;74:321-327.

3. Telford JJ, Carr-Locke DL, Baron TH, et al. A randomized trial comparing uncovered and partially covered self-expandable metal stents in the palliation of distal malignant biliary obstruction. Gastrointest Endosc 2010;72:907-914.

4. Tarantino I, Mangiavillano B, Di Mitri R, et al. Fully covered selfexpandable metallic stents in benign biliary strictures: a multicenter study on efficacy and safety. Endoscopy 2012;44:923-927.

5. Moon SH, Kim MH, Park DH, et al. Modified fully covered selfexpandable metal stents with antimigration features for benign pancreatic-duct strictures in advanced chronic pancreatitis, with a focus on the safety profile and reducing migration. Gastrointest Endosc 2010;72:86-91.

6. Park DH, Lee SS, Lee TH, et al. Anchoring flap versus flared end, fully covered self-expandable metal stents to prevent migration in patients with benign biliary strictures: a multicenter, prospective, comparative pilot study (with videos). Gastrointest Endosc 2011;73:64-70.

7. Park JK, Moon JH, Choi HJ, et al. Anchoring of a fully covered self-expandable metal stent with a $5 \mathrm{~F}$ double-pigtail plastic stent to prevent migration in the management of benign biliary strictures. Am J Gastroenterol 2011;106:1761-1765.

8. Kahaleh M, Tokar J, Conaway MR, et al. Efficacy and complications of covered Wallstents in malignant distal biliary obstruction. Gastrointest Endosc 2005;61:528-533.

9. Isayama H, Kawabe T, Nakai Y, et al. Cholecystitis after metallic stent placement in patients with malignant distal biliary obstruction. Clin Gastroenterol Hepatol 2006;4:1148-1153.

10. Suk KT, Kim HS, Kim JW, et al. Risk factors for cholecystitis after metal stent placement in malignant biliary obstruction. Gastrointest Endosc 2006;64:522-529.

11. Khashab MA, Chithadi KV, Acosta RD, et al; ASGE Standards of Practice Committee. Antibiotic prophylaxis for GI endoscopy. Gastrointest Endosc 2015;81:81-89.

12. Lai NM, Chaiyakunapruk N, Lai NA, O'Riordan E, Pau WS, Saint S. Catheter impregnation, coating or bonding for reducing central venous catheter-related infections in adults. Cochrane Database Syst Rev 2016;3:CD007878. 